\title{
Eficiencia térmica en soldadura de la aleación AA6061-T6 por arco eléctrico indirecto modificado y digitalización de señales de intensidad de corriente ${ }^{(\cdot)}$
}

\author{
R.R. Ambriz*, G. Barrera*, R. García* y V.H. López*
}

\begin{abstract}
Resumen
En el presente trabajo de investigación, se presentan los resultados de la eficiencia térmica en la soldadura de una aleación de aluminio 6061-T6 por medio de la técnica de arco eléctrico indirecto modificado (AEIM) ${ }^{[1]}$. Los valores se encuentran en un rango de 90-94 \% dependiendo de la temperatura de precalentamiento y fueron obtenidos a través de un balance térmico que considera a la energía aportada por el arco eléctrico, la cantidad de masa fundida de los perfiles de soldadura obtenidos y las variables operativas en el proceso de unión, poniendo especial atención en los datos recogidos en el proceso de digitalización para la intensidad de corriente del arco eléctrico. También se emplearon algunos parámetros adimensionales para determinar el grado de aproximación de la porción del baño fundido, el ancho de la zona afectada por el calor (ZAC) y sus correspondientes aproximaciones con los resultados experimentales.
\end{abstract}

Palabras clave Eficiencia térmica; AA6061-T6; Intensidad de corriente; Digitalización; ZAC.

\section{Thermal efficiency on welding of AA6061-T6 alloy by modified indirect electric arc and current signals digitalization}

\begin{abstract}
The results of the thermal efficiency on welding by modified indirect electric arc technique (MIEA) ${ }^{[1]}$ of the 6061 T6 aluminum alloy are presented. These values are in a range of 90 to $94 \%$, which depend of the preheating employed. Thermal efficiency was obtained by means of a balance energy which considers the heat input, the amount of melted mass of the welding profiles, and welding parameters during the joining, especially of the arc current data acquisition. Also, some dimensionless parameters were employed in order to determine the approximation grade of the melted pool, the heat affected zone (HAZ), and their corresponding values with the experimental results.
\end{abstract}

Keywords

Thermal efficiency; AA6061-T6; Current; Data acquisition; HAZ.

\section{INTRODUCCIÓN}

La eficiencia térmica en soldadura por fusión y arco eléctrico está determinada por la cantidad de calor aportado durante la unión y es una función directa del proceso de soldadura empleado y las variables operativas del mismo, entre las cuales se encuentran el voltaje aplicado, la velocidad de avance de la fuente térmica (desplazamiento del cabezal), la intensidad de corriente, la separación entre pieza de trabajo y electrodo o material de aporte. Las eficiencias térmicas para varios procesos de soldadura se determinaron previamente $e^{[2]}$ y se encuentran en un rango amplio, desde baja eficiencia térmica, como el GTAW, hasta los más eficientes como el proceso SAW. Sin embargo, estos valores se han presentado en forma general, considerando las variables operativas del proceso de soldadura en un arco abierto sobre una superficie de trabajo y tomando, como fundamento, la siguiente expresión matemática.

$$
q=\eta \frac{V I}{V}
$$

donde, $q$ es el calor aportado por el arco eléctrico, $\eta$ es la eficiencia térmica, $V$ el voltaje aplicado, I la

\footnotetext{
(•) Trabajo recibido el día 26 de diciembre de 2007 y aceptado en su forma final el día 14 de enero de 2009.

* Instituto de Investigaciones Metalúrgicas, Universidad Michoacana de San Nicolás de Hidalgo, Apdo. Post 888, Morelia, Mich., México. C.P. 58000.ricraf74@gmail.com.
} 
intensidad de corriente y $v$ la velocidad de avance de la fuente térmica.

En el presente trabajo se determina la eficiencia térmica de uniones soldadas empleando la técnica $\left.\mathrm{AEIM}^{[1}{ }^{\mathrm{y}} 3\right]$. Esta técnica de soldadura emplea un proceso semiautomático de soldadura de arco eléctrico, gas de protección y material de aporte (GMAW por sus siglas en ingles), en una aleación Al-Si-Mg (6061T6). Como es conocido, el proceso de soldadura GMAW utiliza una fuente de energía de potencial constante, por lo cual esta variable no representa un factor considerable y puede controlarse fácilmente al igual que la velocidad de avance. Sin embargo, la intensidad de corriente aplicada durante la soldadura, si tiene un efecto importante ya que esta depende de la velocidad de alimentación del material de aporte, de la separación de la boquilla de contacto con la pieza de trabajo y del gas de protección. Estos factores conducen a que los niveles de intensidad de corriente sean distintos y, por lo tanto, la transferencia de metal pueda darse por transferencia globular, spray o cortocircuito ${ }^{[4]}$, provocando discontinuidades poco relevantes, como una mala apariencia (salpicaduras) o defectos importantes que disminuyen las propiedades mecánicas de las uniones (socavados, falta de fusión y/o penetración). Por tal razón, la medición y digitalización de señales de intensidad de corriente durante la soldadura en tiempo real es fundamental para obtener información acerca de los cambios de esta variable y, con ello, determinar de forma más precisa y cuantificada la cantidad de calor aportado, su relación con la masa de material fundido y, por tanto, su eficiencia térmica.

También se abordan algunos aspectos sobre las condiciones mecánicas y microestructurales, que han sido expuestas en trabajos anteriores en soldadura

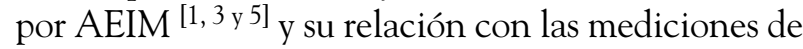
intensidad de corriente y la eficiencia térmica calculada por medio de un balance térmico.

\section{DESARROLLO EXPERIMENTAL}

\subsection{Soldadura}

Se prepararon placas de aluminio 6061-T6 de 12,7 $\mathrm{mm}$ de espesor por $170,0 \mathrm{~mm}$ de longitud y $68,0 \mathrm{~mm}$ de ancho, empleando el tipo de junta que se presenta en la figura 1, la cual permite la aplicación de un

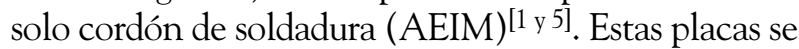
soldaron a través de una máquina semiautomática de corriente directa con una capacidad de $300 \mathrm{~A}$, que emplea el proceso de soldadura GMAW. Se utilizó corriente continua electrodo positivo (CDEP), con un potencial constante de $24 \mathrm{~V}$. El material de

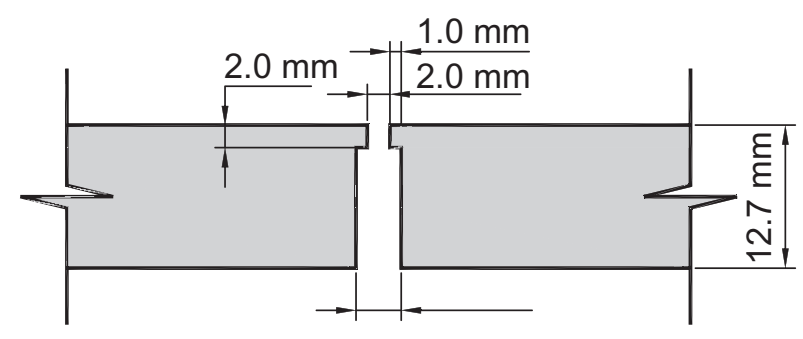

Figura 1. Preparación de junta por AEIM.

Figure 1. Joint preparation for MIEA.

aporte fue un electrodo de alto contenido de silicio (ER4043) con un diámetro de 1,2 mm, el cual fue alimentado a $228 \mathrm{~mm} \mathrm{~s}^{-1}$, con un desplazamiento del cabezal de $3.6 \mathrm{~mm} \mathrm{~s}^{-1}$. El gas de protección fue argón al $100 \%$ con un flujo constante de 23,6 1 $\times \mathrm{min}^{-1}$, mientras que la boquilla de contacto con respecto a las piezas de trabajo se mantuvo a $20 \mathrm{~mm}$. Previamente a la soldadura, las placas se precalentaron a 50,100 y $150^{\circ} \mathrm{C}$.

\subsection{Medición de intensidad de corriente}

La medición de intensidad de corriente se llevó a cabo con un sensor de efecto Hall. La salida del sensor se da en $\mathrm{mV}$ y el rango de operación del transductor va de 0 a 600 Amperios de CD. La relación de voltaje con respecto a la intensidad de corriente es de 1 $\mathrm{mV}=1 \mathrm{~A}$. El sensor se ubicó, directamente, a la salida del polo negativo de la máquina de soldar.

La señal se digitalizó por medio de una tarjeta de adquisición de datos, DAQ, con conexión a puerto USB-2. Se estableció una velocidad de muestreo de 100 lecturas por segundo (conversión de analógico a digital de $100 \mathrm{~Hz}$ ). Para la fase de adquisición de datos, se utilizaron programas elaborados en lenguaje de programación gráfico $\mathrm{G}$, mejor conocido como "Instrumentación Virtual" (LabVIEW 8.2), bajo ambiente del sistema operativo Windows XP.

Se diseñó un dispositivo para identificar el inicio de la medición de intensidad de corriente en las placas a soldar, después de que el arco eléctrico había sido estabilizado por medio de extensiones en las placas a soldar. Este dispositivo consta de un par de láminas de cobre muy delgadas ubicadas al inicio y al final de las placas a soldar, y se colocó una tercera lámina en el cabezal de la máquina. Las laminas inicial y final, en las placas a soldar, se conectaron al negativo de una fuente de potencial constante de $1.5 \mathrm{~V}$, mientras que la lámina en el cabezal, se conecto al positivo de la misma fuente. A su vez, la fuente de voltaje se conectó, en paralelo, al canal 1 de la 
tarjeta de adquisición de datos, de tal forma que el voltaje medido es de $1.5 \mathrm{~V}$.

Una vez iniciado el proceso de soldadura y estabilizado el arco, el cabezal avanza y después de un determinado tiempo la lamina ubicada en este, hace contacto con su correspondiente ubicada al inicio de las placas a soldar, en ese momento el voltaje en el canal 1 es igual a cero, debido al cortocircuito provocado. Se estableció que, a partir de este tiempo de contacto, éstos serian los datos a tomar en cuenta como los que corresponden al inicio de la soldadura. El proceso termina cuando la lámina del cabezal hace contacto con su correspondiente, al final de las placas soldadas. En la figura 2 se ilustra, esquemáticamente, el dispositivo empleado.

\subsection{Perfiles macroestructurales y baño fundido de soldadura}

La figura 3, muestra los perfiles transversales de soldadura obtenidos para cada condición de soldeo ${ }^{[5]}$. Estas secciones transversales fueron reproducidas y trazadas en tres dimensiones por medio de un programa de dibujo asistido por computadora (AutoCAD), considerando un espesor de 3,6 mm, que corresponde a 1 segundo de avance de la fuente térmica o cabezal. El objetivo de trazar los perfiles transversales de soldadura fue cuantificar la cantidad de material fundido (volumen) en un segundo de avance, así como el área y el perímetro. Estos datos se emplearon para el cálculo de la eficiencia térmica en

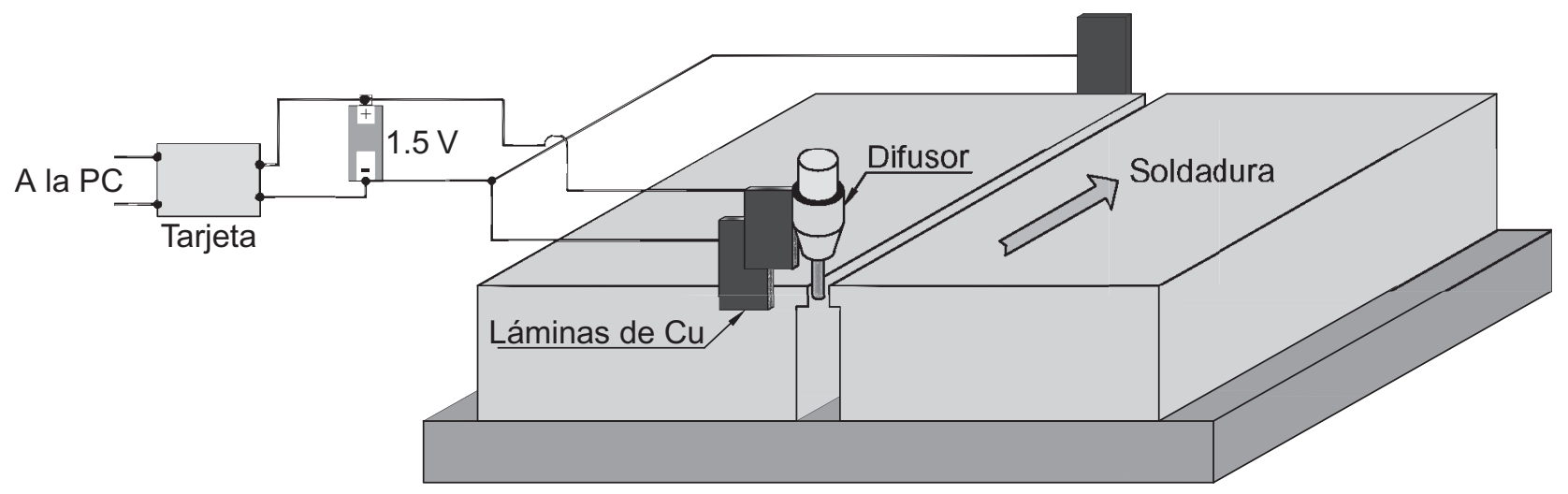

Figura 2. Arreglo para mediciones de intensidad corriente.

Figure 2. Diagram for the current measurements.
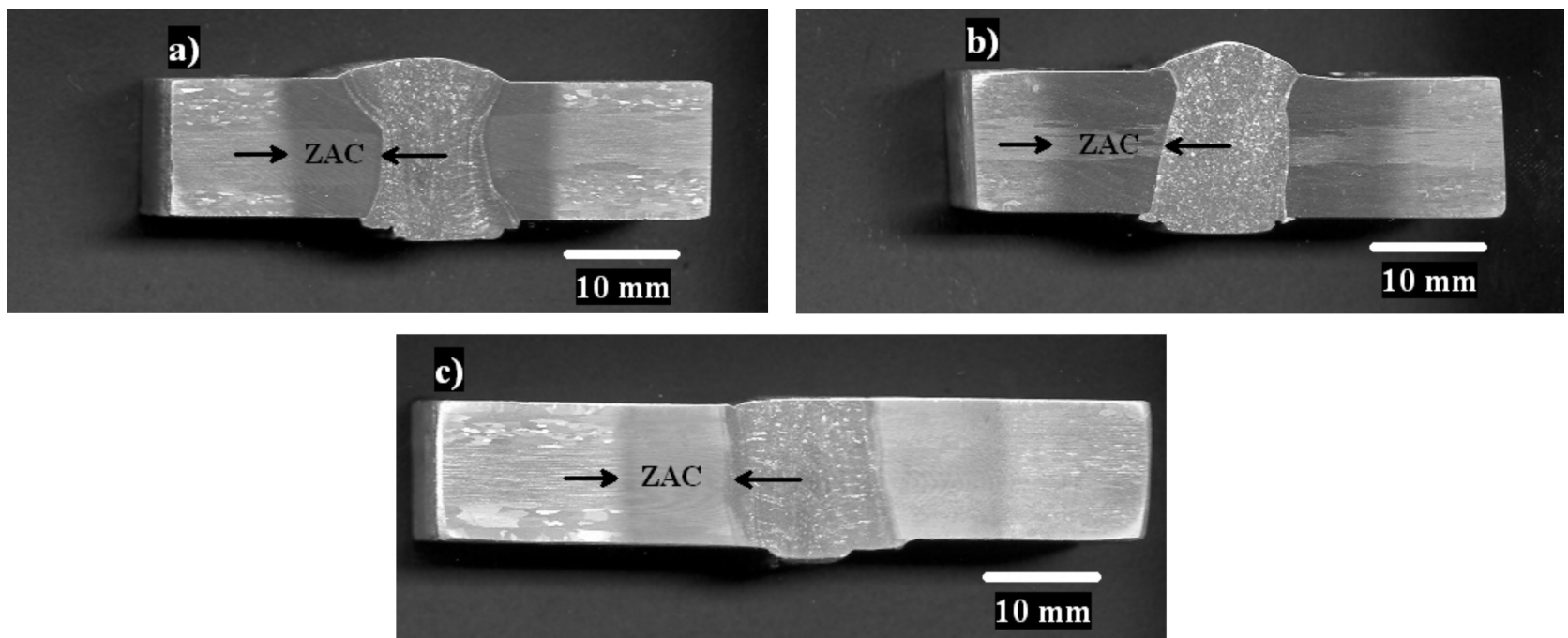

Figura 3. Perfiles de soldadura, a) AEIM $50^{\circ} \mathrm{C}$, b) AEIM $100^{\circ} \mathrm{C}$ y c) AEIM $150^{\circ} \mathrm{C}$.

Figure 3. Welding profiles, a) MIEA $50^{\circ} \mathrm{C}$, b) MIEA $100^{\circ} \mathrm{C}$ and c) MIEA $150^{\circ} \mathrm{C}$. 
conjunto con las dimensiones de la zona afectada por el calor (ZAC), medidas por este mismo medio. Posteriormente, se aplicaron algunas expresiones matemáticas adimensionales que relacionan al ancho de la ZAC en función de las propiedades termofísicas de la aleación y se compararon con las mediciones reales de los perfiles.

\subsection{Balance térmico}

Se realizó un balance térmico de las uniones por AEIM en la aleación 6061-T6, para determinar la eficiencia térmica, en función de la porción de volumen fundido de material base y de aporte en la junta soldada, considerando la cantidad de energía suministrada por el arco eléctrico y aplicando ecuaciones matemáticas fundamentales, como la ley de Fourier para el flujo de calor por conducción en las placas soldadas y la ecuación para la variación de la energía con respecto de la temperatura.

\section{RESULTADOS}

\subsection{Intensidad de corriente del arco eléctrico}

El procesado de la información a partir del rango de datos reales obtenidos en la medición de intensidad de corriente, de acuerdo con el inicio y final del circuito de la figura 2, se llevo a cabo por medio de un programa elaborado en LabVIEW ${ }^{\mathrm{TM}}$, a partir del cual es posible obtener los gráficos de las señales originales y filtradas. La figura 4, presenta un ejemplo del procesado de las señales obtenidas para la soldadura de la aleación 6061-T6 a $50 \stackrel{\circ}{ } \mathrm{C}$ de precalentamiento, en el cual se observan los gráficos correspondientes a la señal original, rango o límite de la señal original, la señal corregida y finalmente la señal filtrada.

Obsérvense los picos de intensidad de corriente que se presentan en el grafico de la señal original durante el proceso de soldadura, los cuales se dan por un espacio de tiempo muy corto (tomando en consideración a la velocidad de muestreo de 100 muestras por segundo, se trata de un tiempo de 1/100 s), sin embargo la mayoría de los datos se encuentran dentro de un rango de 215 y $240 \mathrm{~A}$.

La intensidad de corriente del arco eléctrico en el proceso de soldadura GMAW depende, principalmente, de la velocidad de alimentación del material de aporte (electrodo), el cual es alimentado por un dispositivo mecánico que tiene la función de desenrollar al mismo a través del paso de éste por un sistema de rodillos, por lo cual existe una alta probabilidad de tener variaciones en la velocidad de alimentación que ocasionan inestabilidad del arco eléctrico por periodos cortos y sobresaltos en las mediciones de intensidad de corriente. Estas ligeras variaciones son detectables debido a la alta velocidad con la cual se adquieren los datos.

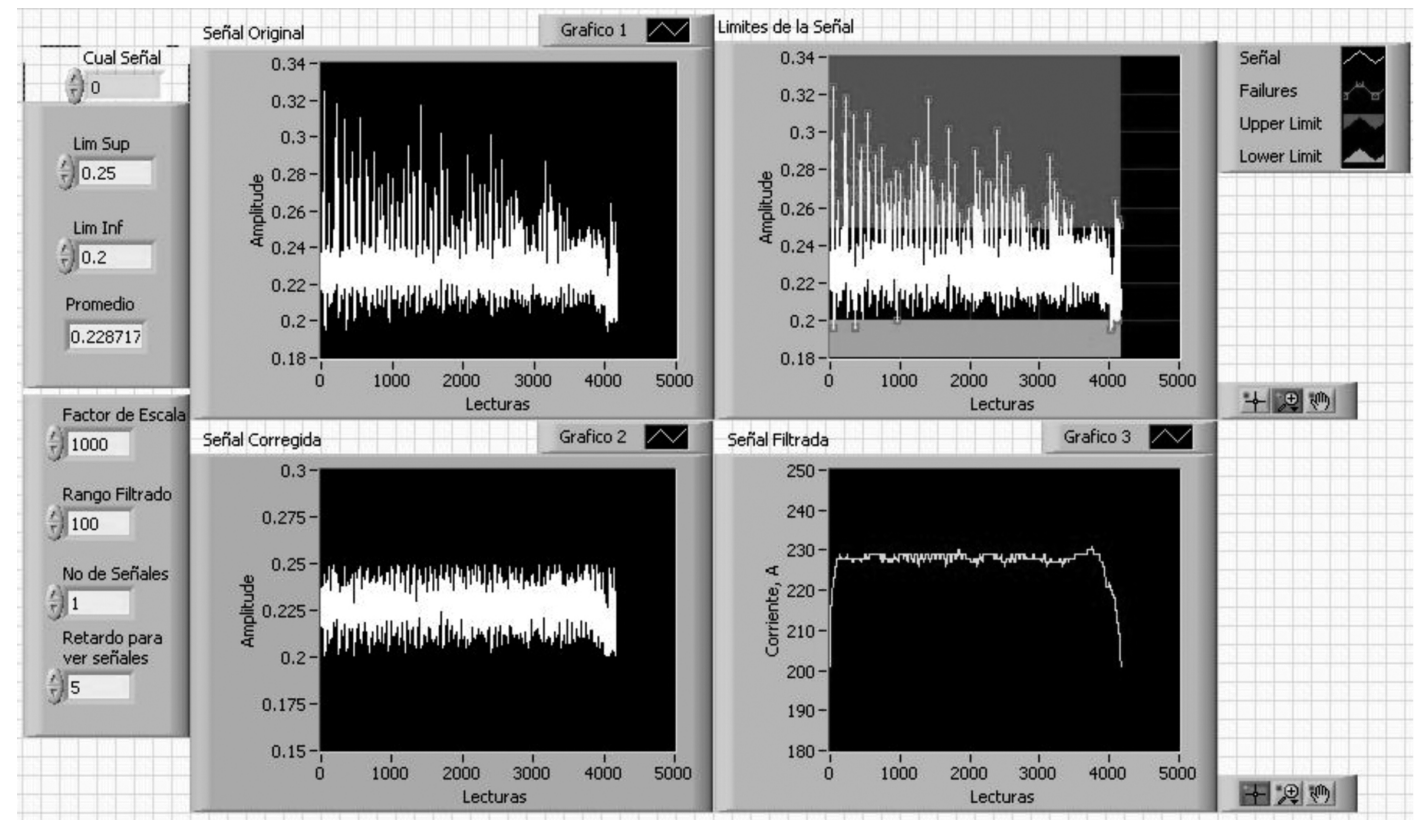

Figura 4. Procesamiento de las señales de intensidad de corriente del arco eléctrico.

Figure 4. Processing of the electric arc current signals. 
Partiendo de la señal original se determina, entonces, el promedio, la desviación estándar y la moda para los datos adquiridos. En virtud de que las variaciones de intensidad de corriente, detectadas a lo largo del proceso de soldadura, resultan ser no relevantes, debido a que no se presentaron discontinuidades (defectos) importantes atribuibles a éstas, en las juntas soldadas (Fig. 5), se tomó la determinación de corregir a la señal original. El algoritmo para llevar a cabo la corrección consistió en dos fases: en una primera, los valores que sobrepasaron un determinado rango preestablecido entre 200 y $250 \mathrm{~A}$, se llevaron al valor promedio calculado de la señal original. En la segunda fase, la señal corregida se pasó por un filtrado tipo medio para un rango determinado de los valores de entrada y, así se obtuvo, finalmente, la señal filtrada (Fig. 4). La tabla I, presenta los valores de medición de intensidad de corriente.

El grafico de medición de intensidad de corriente se presenta en la figura 6 y, en él, se aprecia que, prácticamente, no existe una variación importante con respecto de la temperatura de precalentamiento, lo que era de esperar debido a que, ésta, no depende de la temperatura de las placas a soldar. Aquí, hay que destacar que la señal de intensidad de corriente cuando la temperatura de precalentamiento fue de $150^{\circ} \mathrm{C}$ se encuentra ligeramente por arriba de las otras dos, lo que significó una mayor cantidad
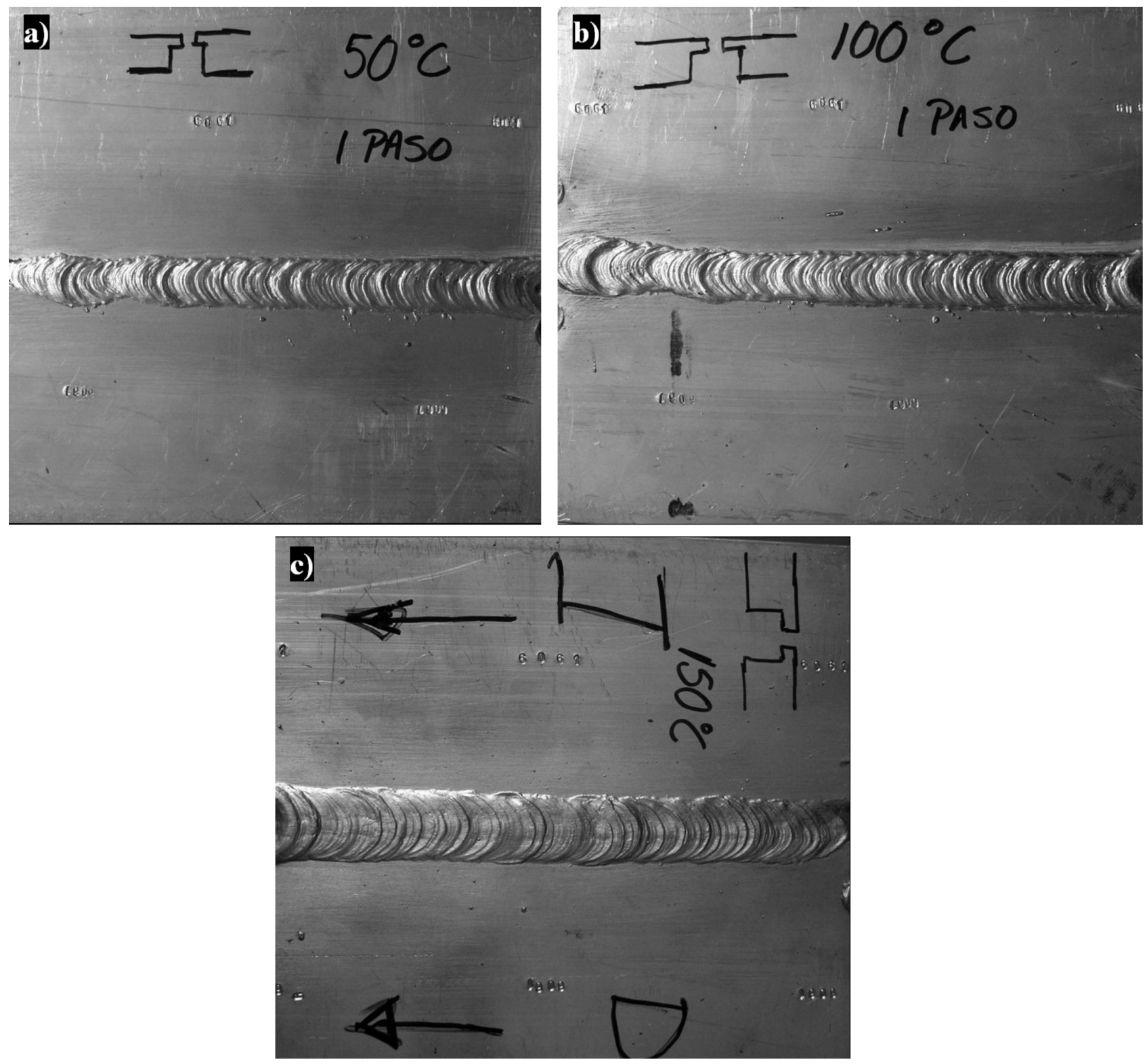

Figura 5. Apariencia de los cordones de soldadura en la aleación 6061-T.

Figure 5. Images of the weld beads on the 6061-T6 alloy. 
Tabla I. Mediciones de intensidad de corriente

Table I. Current measurements

\begin{tabular}{cccc}
\hline Precalentamiento $\left({ }^{\circ} \mathrm{C}\right)$ & \multicolumn{3}{c}{ Intensidad de Corriente $(\mathrm{A})$} \\
\cline { 2 - 4 } & Promedio & Moda & Desviación Est. \\
\hline 50 & 228,72 & 259,48 & 13,16 \\
100 & 227,84 & 237,77 & 13,12 \\
150 & 232,94 & 255,93 & 15,89 \\
\hline
\end{tabular}

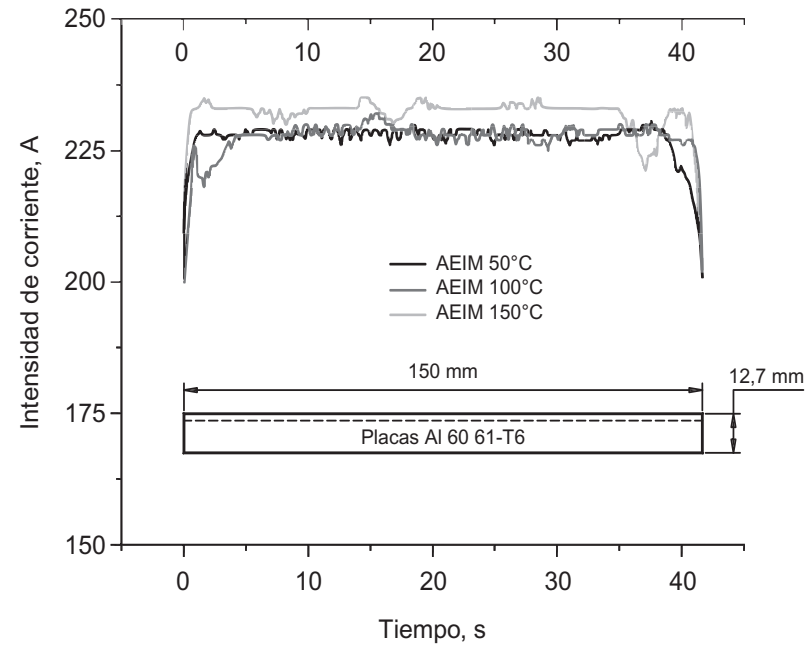

Figura 6. Grafico de intensidad de corriente para 6061-T6 a lo largo de las placas soldadas.

Figure 6. Current graph for 6061-T6 along of the welded plates. de calor aportado durante la soldadura y, por tanto, es de esperar que, bajo estas condiciones, se tenga la capacidad de fundir una mayor cantidad de material.

\subsection{Perfiles macroestructurales y baño fundido de soldadura}

El volumen del baño fundido, es un indicativo importante ya que, en función de la cantidad de material fundido, se puede determinar si las condiciones bajo las cuales se realizan las soldaduras son capaces de llenar el espacio de la preparación de junta (Fig. 1), el cual tiene un volumen de $168,8 \mathrm{~mm}^{3}$, considerando un espesor de $3,6 \mathrm{~mm}$, correspondiente a un segundo de avance del cabezal.

La figura 7, muestra los perfiles en tres dimensiones de las porciones fundidas después del proceso de soldadura. Tanto el perfil, el área de la sección transversal, el perímetro, dimensiones de la ZAC y el volumen fundido en función de la velocidad de desplazamiento,
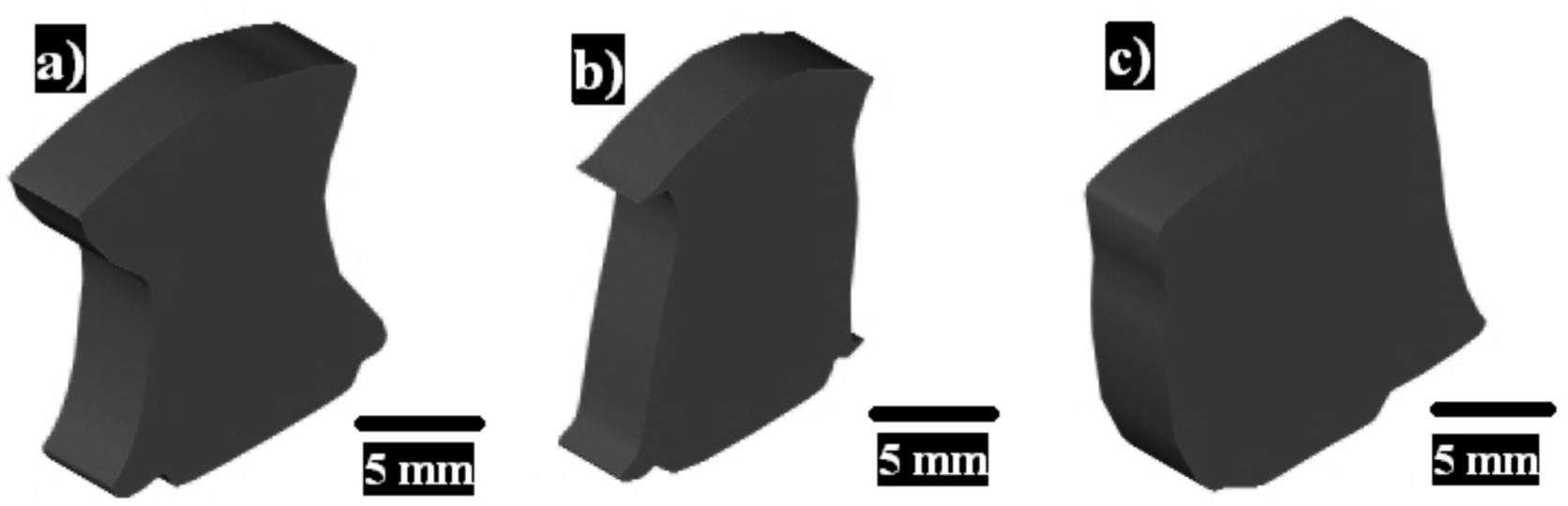

Figura 7. Perfiles en tres dimensiones de la zona de fusión.

Figure 7. Three dimensional profiles of the fusion zone. 
fueron obtenidos por medio de un software de diseño asistido por computadora (AutoCAD), tomando en consideración las secciones transversales reales de las soldaduras (Fig. 3). La tabla II, presenta los resultados correspondientes.

Empleando las variables operativas de las soldaduras y las mediciones de intensidad de corriente presentadas anteriormente, los resultados experimentales en cuanto a dimensiones fueron analizados y comparados con algunos cálculos teóricos, mediante relaciones matemáticas adimensionales ${ }^{[6]}$. Estas relaciones están representadas en la figura 8 [7], por medio de las siguientes expresiones:

$$
\begin{gathered}
n=\frac{\eta q v}{4 \pi \alpha^{2}\left(H_{c}-H_{0}\right)} \\
\theta=\frac{T-T_{0}}{T_{c}-T_{0}} \\
V o l=\frac{8 \alpha^{3}}{V^{3}} \Gamma \\
\Gamma=\frac{\pi}{12}\left[3\left(\frac{n}{\theta}\right)^{2}-3\left(\xi^{\prime}\right)^{2}-4\left(\xi^{\prime}\right)^{3}\right] \\
\xi^{\prime}=\frac{1}{2} \ln \left(\frac{n / \theta}{\xi^{\prime}}\right) \\
\Lambda_{1}=A \frac{v^{2}}{4 \alpha^{2}}
\end{gathered}
$$

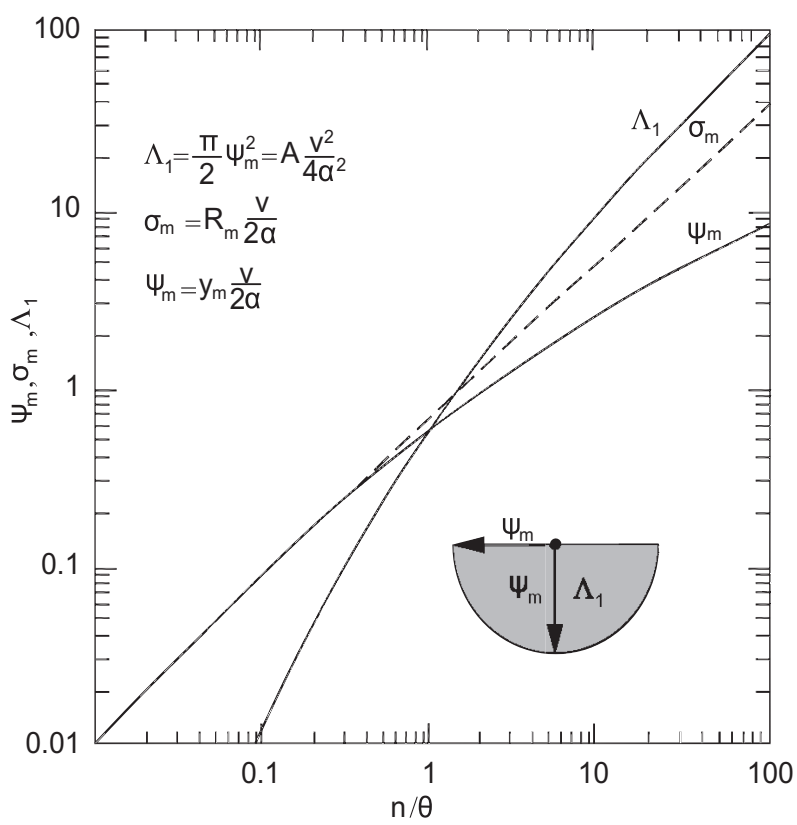

Figura 8. Parámetros adimensionales de operación, de distancia, ancho y sección transversal.

Figure 8. Dimensionless parameters of operation, distance, width, and transversal section.

$$
\Psi_{m}=y_{m} \frac{v}{2 \alpha}
$$

La tabla III, presenta el significado de los símbolos empleados en las ecuaciones anteriores. Tomando en consideración la eficiencia térmica del proceso de soldadura GMAW reportado por H. B. Smart ${ }^{[8]}$ como una primera aproximación, aplicando las ecuaciones (2) y (3) y por medio de las relaciones adimensionales de operación y de temperatura $n / \theta$, (Fig. 8), se determinó el volumen del baño fundido de soldadura y la relación con el ancho de la ZAC a una temperatura de referencia, correspondiente a la transformación

Tabla II. Mediciones experimentales para las geometrías de las juntas soldadas

Table II. Experimental measurements for the geometries of the welded joints

\begin{tabular}{cccccc}
\hline Precalentamiento $\left({ }^{\circ} \mathrm{C}\right)$ & $\begin{array}{c}\text { Perímetro } \\
\text { Fundido }(\mathrm{mm})\end{array}$ & $\begin{array}{c}\text { Area } \\
\text { Fundida }\left(\mathrm{mm}^{2}\right)\end{array}$ & $\begin{array}{c}\text { Volumen } \\
\text { Fundido* }\left(\mathrm{mm}^{3} \mathbf{s}^{-1}\right)\end{array}$ & $\begin{array}{c}\text { Ancho ZAC } \\
(\mathrm{mm})\end{array}$ & $\begin{array}{c}\text { Area ZAC } \\
\left(\mathrm{mm}^{2}\right)\end{array}$ \\
\hline 50 & 61,90 & 181,4 & 653,0 & 6,50 & 158,6 \\
100 & 61,20 & 185,9 & 669,4 & 8,70 & 226,4 \\
150 & 54,30 & 187,6 & 677,8 & 10,3 & 254,4 \\
\hline
\end{tabular}

* Considerando la velocidad de desplazamiento del cabezal $\left(3.6 \mathrm{mms}^{-1}\right)$. 
Tabla III. Lista de símbolos empleados en ecuaciones y relaciones adimensionales

Table III. Symbols employed for the dimensionless relations and equations

\begin{tabular}{ll}
\hline Símbolo & \multicolumn{1}{c}{ Definición y Unidades } \\
\hline$H_{c}-H_{o}$ & Calor por unidad de volumen, referida al punto de fusión, $\mathrm{J} \mathrm{mm}^{-3}$ \\
$k$ & Intensidad de corriente, $\mathrm{A}$ \\
$q$ & Conductividad térmica, $\mathrm{J} \mathrm{s}^{-1} \mathrm{~mm}^{-1}{ }^{\circ} \mathrm{C}^{-1}$ \\
$T$ & Calor aportado por el arco eléctrico, $\mathrm{Js}^{-1}$ \\
$T_{c}$ & Temperatura, ${ }^{\circ} \mathrm{C}$ \\
$T_{f}$ & Temperatura de referencia, ${ }^{\circ} \mathrm{C}$ \\
$T_{o}$ & Temperatura de fusión, ${ }^{\circ} \mathrm{C}$ \\
$T_{L}$ & Temperatura de precalentamiento de la placa, ${ }^{\circ} \mathrm{C}$ \\
$V$ & Temperatura de líquido, ${ }^{\circ} \mathrm{C}$ \\
$V o l$ & Voltaje del arco eléctrico, $\mathrm{V}$ \\
$V$ & Volumen de la pileta líquida de soldadura, $\mathrm{mm}^{3}$ \\
$y_{m}$ & Velocidad de avance del arco eléctrico, mm $\mathrm{s}^{-1}$ \\
$\alpha$ & Ancho de la zona afectada térmicamente, $\mathrm{mm}^{-1}$ \\
$\Delta H_{f}$ & Difusividad térmica, mm ${ }^{2} \mathrm{~s}^{-1}$ \\
$\eta$ & Calor latente de fusión, $\mathrm{J}$ gmol ${ }^{-1}$ \\
$\Lambda_{1}$ & Eficiencia térmica, \% \\
$\theta$ & Parámetro adimensional de la sección transversal isotérmica \\
$\xi$ & Parámetro adimensional de temperatura pico \\
$\psi_{\mathrm{m}}$ & Distancia adimensional hacia el frente de la fuente térmica en $x$ \\
\hline
\end{tabular}

líquido-sólido de la aleación. Los datos empleados fueron: $\alpha=62 \mathrm{~mm}^{2} \mathrm{~s}^{-1}, H_{c}-H_{0}=1.71 \mathrm{Jmm}^{-3}, T_{c}=582^{\circ} \mathrm{C}$ y $T_{f}=652^{\circ} \mathrm{C}$. El volumen del baño fundido de soldadura está determinado por la ecuación (4), la cual está en función del parámetro adimensional de volumen $\Gamma$, determinado por la ecuación (5) y esta, a su vez, hace referencia a la distancia adimensional $\xi^{\prime}$, a partir de la fuente térmica hacia el frente de la isoterma del baño de soldadura, representado por la ecuación (6). La representación esquemática de estas relaciones adimensionales se muestra en la figura 9. Los resultados teóricos para el volumen del baño fundido de soldadura se presentan en la tabla IV.

El área de la sección transversal del baño fundido puede determinarse, entonces, a partir del valor obtenido del parámetro adimensional para el área de la sección transversal $\Lambda_{1}$, calculado a partir de los valores obtenidos para la relación $n / \theta$ y aplicando la ecuación (7). De la misma forma se puede calcular, aproximadamente, el ancho de la ZAC, $y_{m}$, tomando en consideración la temperatura pico de transformación microestructural de $400 \stackrel{\circ}{\mathrm{C}}$, empleando la ecuación (8), la cual es una función del valor de la coordenada adimensional, $\Psi_{m}$, representada en la figura 9 , de la velocidad de avance y de la difusividad térmica, $\alpha$. Los resultados se presentan en la tabla $\mathrm{V}$.

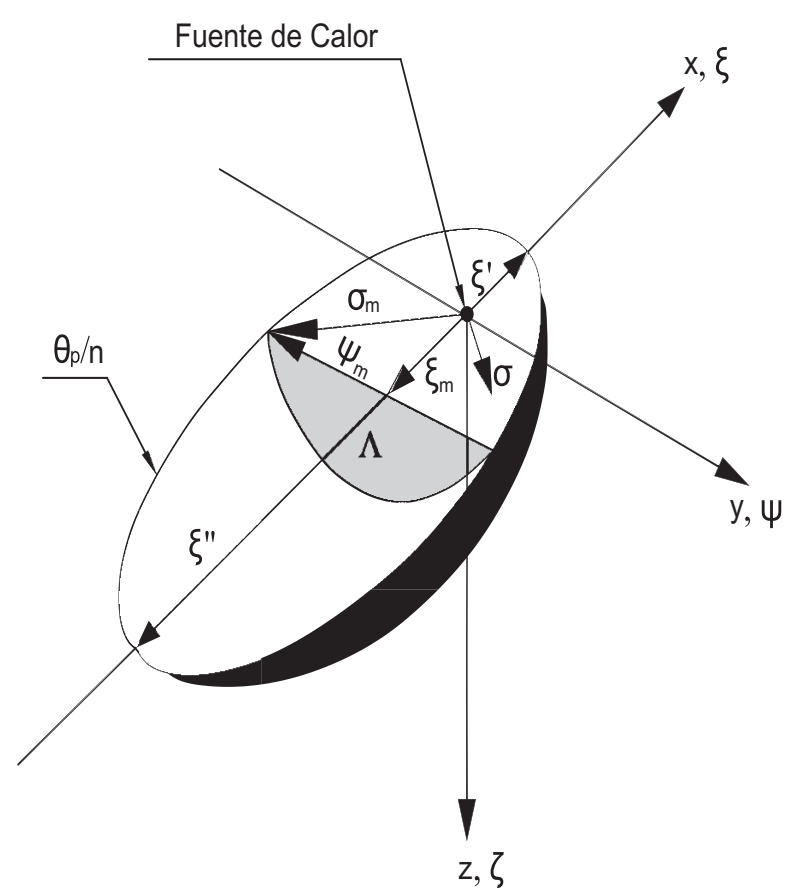

Figura 9. Representación esquemática del baño fundido de soldadura.

Figure 9. Schematic representation of the weld pool. 
Tabla IV. Volumen del baño fundido de soldadura

Table IV. Volume of the weld pool melted

\begin{tabular}{rrrr}
\hline $\begin{array}{c}\text { Temp. } \\
\text { Prec. }\left({ }^{\circ} \mathrm{C}\right)\end{array}$ & $\xi^{\prime} \times 10^{3}$ & $\Gamma \times 10^{3}$ & $\mathrm{Vol}\left(\mathrm{mm}^{3}\right)$ \\
\hline 50 & 153,2 & 11,45 & 467,91 \\
100 & 154,0 & 11,92 & 487,11 \\
150 & 158,0 & 13,24 & 541,06 \\
\hline
\end{tabular}

Tabla V. Área de la sección transversal del baño fundido y ancho de la ZAC

Table $V$. Transversal section area of the melted pool and HAZ width

\begin{tabular}{ccccc}
\hline $\begin{array}{c}\text { Temp. } \\
\text { Prec. }\left({ }^{\circ} \mathrm{C}\right)\end{array}$ & $\Lambda_{1} \times 10^{3}$ & $\Psi_{\mathrm{m}} \times 10^{3}$ & $\mathrm{~A}\left(\mathrm{~mm}^{2}\right)$ & $\mathrm{y}_{\mathrm{m}}(\mathrm{mm})$ \\
\hline 50 & 48,30 & 250 & 57,30 & 8,61 \\
100 & 51,25 & 270 & 60,80 & 9,30 \\
150 & 60,00 & 290 & 71,18 & 9,98 \\
\hline
\end{tabular}

Obsérvese que el volumen del baño fundido siempre es mayor al espacio vacío por llenar, aspecto que beneficia para la aplicación de una sola pasada de soldadura, además de que el aprovechamiento de la energía que es perdida por radiación y convección en la soldadura, cuando el arco se encuentra expuesto a la atmósfera (soldadura por pasos múltiples), favorece también al proceso por AEIM ocasionando que, finalmente, se obtenga una cantidad de masa fundida considerable (Fig. 3 y Tabla IV) y, por lo tanto, el proceso de soldadura tienda a ser más eficiente.
Un aspecto a considerar, al momento de establecer las variables operativas para la realización de las soldaduras, es el efecto que tendrá el calor aportado sobre el ancho de la ZAC transformada, debido a los cambios microestructurales de la aleación 6061-T6 cuando, esta, se somete a gradientes térmicos por encima de la temperatura de envejecimiento artificial, que producen un descenso drástico en propiedades mecánicas de las uniones soldadas $\left.{ }^{[5} \mathrm{y} 9\right]$, motivado por la formación de una zona de baja dureza y la tendencia al agrietamiento por licuación que se ocasiona por la difusión de elementos de bajo punto de fusión hacia la ZAC ${ }^{[10-12] . ~}$

Tales cambios microestructurales son atribuidos a la transformación de los precipitados incoherentes, $\beta^{\prime \prime}$, en los coherentes, $\beta^{\prime}$, de acuerdo con la secuencia de precipitación establecida por Dutta y Allen ${ }^{[13]}$. Uno de los objetivos de este trabajo de investigación es observar los resultados experimentales y teóricos para los anchos de la ZAC, con la intención de que estos puedan ser tomados como referencia y relacionados con las propiedades mecánicas obtenidas en las uniones por AEIM.

\subsection{Eficiencia térmica en las uniones por AEIM}

La energía generada por el arco eléctrico en los procesos de soldadura se aprovecha de dos formas: una, que es la energía necesaria para fundir al metal de aporte y al metal base y, otra, que es la energía que se disipa por conducción a través del material soldado. En el caso de las soldaduras por AEIM, la forma en la cual se disipa el calor por conducción es en dos dimensiones (Fig. 10), debido a que estas son de penetración completa y de una sola pasada de soldadura.

DuPont y Marder ${ }^{[2]}$, establecieron un esquema para el balance de energía con el fin de determinar la

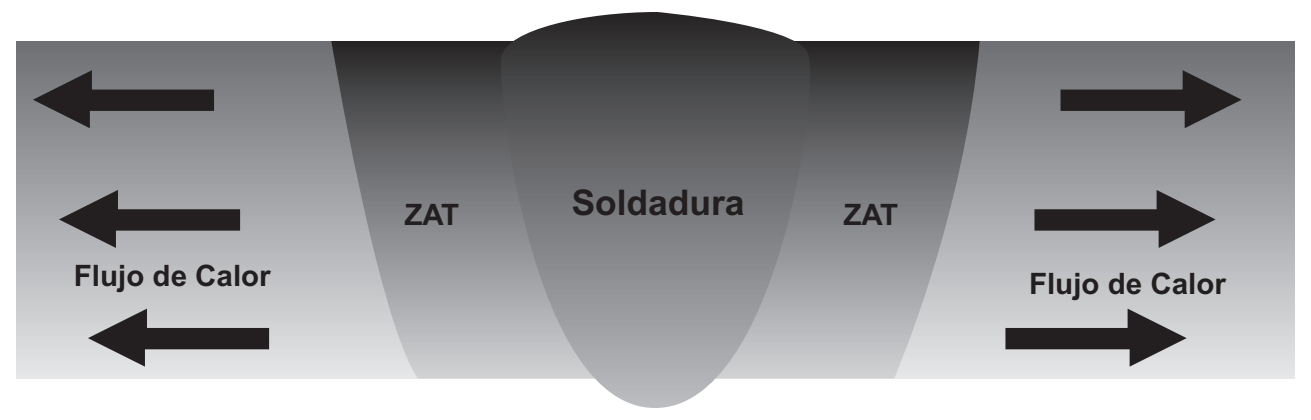

Figura 10. Flujo de calor por conducción en soldadura por AEIM.

Figure 10. Conduction heat flow in MIEA welds. 
eficiencia térmica de los procesos de soldadura por arco eléctrico, de acuerdo con la figura 11. Puede observarse que una parte de la energía generada por el arco eléctrico no es aprovechada, debido a que se pierde por convección y radiación a través de la columna del arco eléctrico, lo que ocasiona una disminución de la eficiencia térmica de los procesos de soldadura.

La finalidad del presente balance térmico, es determinar la eficiencia térmica en la soldadura por AEIM en aleaciones de aluminio, en función de la masa de material fundido. En la figura 11 se muestra de forma esquemática el efecto de la energía suministrada sobre la fusión del material base y de aporte. El calor aportado por unidad de tiempo (potencia calorífica) en los procesos de soldadura depende, fundamentalmente, de las variables operativas del voltaje, $V$, y la intensidad de corriente, $I$, de acuerdo con la ecuación (9).

$$
\text { Calor aportado }=\text { VI }
$$

El calor necesario para la fusión de un volumen determinado de masa metálica, está dado por la ecuación para la variación de la energía con respecto a la temperatura. Además, debe considerarse al calor latente de fusión del metal líquido, $\mathrm{C}_{p}$, por lo cual la ecuación que expresa de forma correcta la variación de la energía, , entre los límites de temperatura inicial $T_{i}$ y temperatura final $T_{f}$ es:

$$
\Delta H=\int_{T_{i}}^{T_{f}} C_{p} d T+\Delta H_{f}
$$

Por medio de un balance de energía, tomando en consideración el calor generado por el arco eléctrico durante la soldadura como única fuente de suministro de energía al sistema y despreciando el efecto de la convección y radiación de la columna del arco eléctrico, se tiene la siguiente expresión:

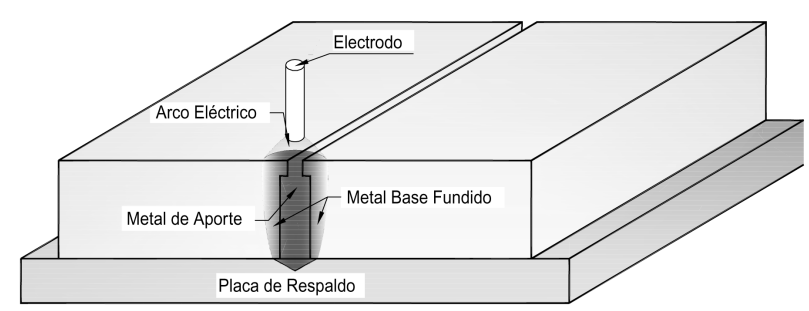

Figura 11. Energía suministrada durante la soldadura en AEIM.

Figure 11. Input energy during the MIEA welding.

\section{Calor aportado por el arco $=$ Variación de la energía + Calor disipado por conducción}

En base a la expresión anterior, es posible plantear la relación general que determinará la eficiencia del proceso de soldadura por AEIM, mediante la siguiente ecuación:

$$
\eta V I=\left[\int_{T_{i}}^{T_{f}} C_{p} d T+\Delta H_{f}\right]+k\left[\frac{\partial^{2} T}{\partial x^{2}}+\frac{\partial^{2} T}{\partial y^{2}}+\frac{\partial^{2} T}{\partial z^{2}}\right]
$$

La ecuación anterior expresa, de forma general, un balance de energía, por lo que deberán considerarse los términos necesarios para homogeneizar a la ecuación, tales como densidad, cantidad de material fundido, peso molecular del metal y área de transferencia de calor por conducción a partir de la interfase sólido-líquido generada por la zona de fusión. La expresión (15) presenta, de forma particular, la expresión para la eficiencia térmica por AEIM en aluminio.

De acuerdo con la termodinámica clásica, la variación del calor específico en un rango de temperaturas puede expresarse mediante una serie de potencias del tipo:

$$
C_{p}=a+b T+c T^{2}
$$

Utilizando el programa termodinámico FACT para encontrar la solución de la serie de potencias para aluminio, en un rango de temperaturas de 720 a $3000 \mathrm{~K}$, se obtiene lo siguiente:

$$
C_{p}=31.751-8.7807673 \times 10^{-12} T^{-2}
$$

Realizando los cálculos necesarios y considerando que el calor latente de fusión del aluminio líquido es $\Delta H_{f}=8522.53 \mathrm{~J} \mathrm{gmol}^{-1}$, y que el calor disipado por conducción puede determinarse suponiendo que esté se pierde a través de cierta longitud de las placas soldadas y una parte por la placa de respaldo, se puede determinar la eficiencia térmica del proceso de soldadura por AEIM de la siguiente manera:

$$
\begin{gathered}
\eta V I=\left\{\left[31.751\left(T_{L}-T_{i}\right)+8.78 \times 10^{-12}\left(T_{L}^{-1}-T_{i}^{-1}\right)\right]+\Delta H_{f}\right\} \frac{\rho Q}{M}+ \\
\\
{\left[2 k_{A l} A_{T} \frac{T_{L}-T_{P}}{L_{T}}+k_{\text {Acerd }} A_{B} \frac{T_{L}-T_{\text {amb }}}{L_{B}}\right]}
\end{gathered}
$$


EFICIENCIA TÉRMICA EN SOLDADURA DE LA ALEACIÓN AA6061-T6 POR ARCO ELÉCTRICO INDIRECTO MODIFICADO Y DIGITALIZACIÓN DE SEÑALES DE INTENSIDAD DE CORRIENTE THERMAL EFFICIENCY ON WELDING OF AA6061-T6 ALLOY BY MODIFIED INDIRECT ELECTRIC ARC AND CURRENT SIGNALS DIGITALIZATION

Tabla VI. Eficiencia térmica en soldaduras por AEIM

Table VI. Thermal efficiency of the MIEA welds

\begin{tabular}{rcccccc}
\hline Prec. $\left({ }^{\circ} \mathrm{C}\right)$ & $\begin{array}{c}\text { Calor de } \\
\text { Fusión }\left(\mathrm{Js}^{-1}\right)\end{array}$ & $\begin{array}{c}\text { Calor perdido } \\
\text { por Conducción } \\
\left(\mathrm{J} \mathrm{s}^{-1}\right)\end{array}$ & $\begin{array}{c}\text { Calor perdido } \\
\text { en placa de } \\
\text { respaldo }\left(\mathrm{J} \mathrm{s}^{-1}\right)\end{array}$ & $\begin{array}{c}\text { Calor } \\
\text { aportado } \\
\left(\mathrm{J} \mathrm{s}^{-1}\right)\end{array}$ & $\begin{array}{c}\text { Masa } \\
\text { fundida } \\
\left(\mathrm{g} \mathrm{s}^{-1}\right)\end{array}$ & $\begin{array}{c}\text { Eficiencia } \\
\text { térmica } \\
(\%)\end{array}$ \\
\hline 50 & $1.791,66$ & $1.954,16$ & $1.009,65$ & $5.260,1$ & 1,76 & 90,4 \\
100 & $1.729,00$ & $1.734,50$ & $1.365,43$ & $5.239,4$ & 1,81 & 92,8 \\
150 & $1.643,78$ & $1.326,46$ & $2.048,14$ & $5.356,7$ & 1,83 & 94,6 \\
\hline
\end{tabular}

donde, $\rho$ es la densidad del aluminio, $Q$ es la cantidad de masa fundida por unidad de tiempo y $\mathrm{M}$ es el peso molecular del aluminio.

La tabla VI, presenta los resultados correspondientes. Nótese como la eficiencia térmica del proceso de soldadura por AEIM, se encuentra por encima de los valores estudiados para un proceso de soldadura GMAW convencional ${ }^{[2]}$. Además, debe observarse que la eficiencia térmica en AEIM se incrementa en función de la temperatura de precalentamiento, la cual es favorable para la obtención de una mayor cantidad de material fundido que beneficia a las características de las soldaduras en cuanto al aprovechamiento de la energía suministrada y, por tanto, un menor grado de afectación en sus propiedades mecánicas finales, tal como ha sido expuesto previamente ${ }^{[1}$ y 5$]$.

\section{CONCLUSIONES}

- La medición de intensidad de corriente del arco eléctrico en tiempo real, durante el proceso de soldadura por AEIM, es un parámetro muy importante en función del calor aportado en la soldadura de aleaciones de aluminio ya que, en función de ello, se puede determinar, de forma teórica mediante relaciones matemáticas adimensionales, la cantidad de material fundido que resulte adecuada para llenar el espacio de junta a soldar, así como una aproximación con la zona afectada por el calor que puede relacionarse, directamente, con las propiedades mecánicas de las uniones.
- La capacidad de aprovechamiento de energía aportada por el arco eléctrico en la técnica de soldadura por AEIM, permite obtener una cantidad de masa fundida que tiende a incrementar la eficiencia térmica en las juntas soldadas de la aleación de aluminio 6061-T6.

\section{REFERENCIAS}

[1] R. R. Ambriz, G. Barrera y R. García, S\&I 11 (2006) 10-17.

[2] J. N. DuPont y A. R. Marder, Weld. J. (1995) 406s-416s.

[3] R. R. Ambriz, G. Barrera, R. García y V. H. López, S\&I 13 (2008) 255-263.

[4] L. A. Jones, T. W. Eager y J.H. Lang, Weld. J. 77 (1998) 135.

[5] R. R. Ambriz, G. Barrera, R García y V. H. Lopez, J. Mat. Design 30 (2009) 2.446-2.453.

[6] O. O. Grong, Metallurgical Modelling of Welding, The Institute of Materials, 1997, p. 7-30.

[7] O. R. Myhr, O. Grong, Acta Metall. 38 (1990) 449-460.

[8] H. B. Smart, Arc welding processes. Welding theory and practice, Elsevier Sci., 1990, pp. 175-188.

[9] V. Malin, Weld. J. 74 (1995) 305s-318s.

[10] N. F. Gittos y M. H. Scott, Weld. J. (1981) 96s$103 \mathrm{~s}$.

[11] T. Enjo y T. Kuroda, Trans. JWRI 11 (1982) 61-66.

[12] C. Huang y S. Kou, Weld. J. (2004) 111s-122s.

[13] I. Dutta y S. M. Allen, J. Mater. Sci. Lett. 10 (1991) 323-326. 